\section{SAGM blood}

Sir,

In reply to the letter by Roberton and Chiswick, ${ }^{1}$ doctors in Sweden probably have the most experience of using SAGM blood. If it is to be used for exchange transfusion they recommend storage for less than five days followed by centrifugation and replacement of the supernatant with frozen AB serum. When reconstituted this provides about 4-5 $\mathrm{mg} / \mathrm{kg}$ body weight of adenine in each exchange transfusion. They have reported excellent results in neonates. For 'top up' transfusions they use red cells in SAGM, estimating that $10 \mathrm{ml}$ will supply $0.57 \mathrm{mg} / \mathrm{kg}$ body weight of adenine. This they consider a safe concentration that will be metabolised between transfusions. They have no evidence of complications associated with mannitol (C F Hogman, personal communication).

Dr $\mathbf{N}$ Luban presented the results of some cases at a workshop on paediatric transfusion organised by the American Association of Blood Banks in 1985, and described crystals found at necropsy in the kidneys of two babies who had received citrate phosphate dextrose adenine blood. Whether these crystals were in fact 2.8 dihydroxyadenine and whether they had caused any renal damage was not clear.

Kreuger ${ }^{2}$ suggested that the kinetics of adenine metabolism are safe if citrate phosphate dextrose adenine blood is used for exchange transfusions. Despite the lack of evidence to suggest that SAGM blood may cause problems in neonates, we continue to use citrate phosphate dextrose as the anticoagulant in our practice.

\section{References}

1 Roberton NRC, Chiswick ML. Concern over safety of SAGM blood. Arch Dis Child 1987;62:638.

2 Kreuger A. Adenine metabolism during and after exchange transfusions in newborn infants with CPD-adenine blood. Transfusion 1976;16:249-252.

K P Windebank Graduate School of Medicine, and S B MOORE Blood Bank and Transfusion Services, Mayo Clinic, Rochester, Minnesota 55905, USA

\section{Glycosylated haemoglobin in cystic fibrosis}

\section{Sir,}

In their letter Caiger et al reported on glycosylated haemoglobin concentrations in children with cystic fibrosis. ${ }^{1}$ They concluded that this measurement could be of value in the early detection and treatment of associated diabetes mellitus in such patients. Bistritzer et $a l^{2}{ }^{2}$ however, reported significantly higher glycosylated haemoglobin concentrations in children with cystic fibrosis than in control subjects even in the absence of documented glucose intolerance. Most of these children were on long term antibiotic treatment.

We would like to draw attention to the interference in glycosylated haemoglobin estimations by penicilloylated haemoglobin. We became aware of this interference when we measured the extent of haemoglobin glycosylation both indirectly by chromatography as haemoglobin $A_{1}$ and specifically by the thiobarbituric acid method in children with cystic fibrosis. (Flückiger $R$, Mathews W, unpublished observations, presented at US National Institute of Health Conference 'Biological mechanisms in aging', June 1980).

The mean glycosylated haemoglobin concentration in children with cystic fibrosis but without diabetes was $9 \%$ compared with $7 \%$ in control subjects. In contrast, thiobarbituric acid values converted for comparison to glycosylated haemoglobin equivalents were $8 \%$ in children with cystic fibrosis and $7 \%$ in controls. The usual association between these two indices of haemoglobin glycosylation was apparent from the data for children with cystic fibrosis and overt diabetes but not for those without glucose intolerance (figure). Examination of the clinical records of the children with raised glycosylated haemoglobin concentrations and normal thiobarbituric acid values showed that these children had all been receiving long term treatment with high doses of beta lactam antibiotics. In vitro incubation of haemoglobin with beta lactams leads to covalent attachment of the penicilloyl moiety to haemoglobin and causes glycosylated haemoglobin mobility of the respective haemoglobins. Direct identification of the penicilloyl moiety in the glycosylated haemoglobin fraction from children with cystic fibrosis was unsuccessful because of the small amounts of penicilloylated haemoglobin.

We conclude that glycosylated haemoglobin measurements can be misleading in children with cystic fibrosis receiving treatment with penicillin. In these patients determination of haemoglobin glycosylation by specific

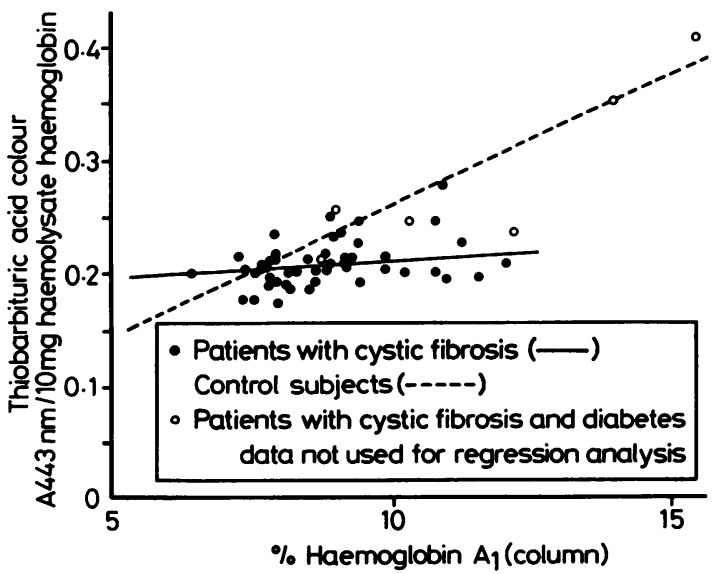

Figure Glycosylated haemoglobin measured by chromatography and by thiobarbituric acid method in patients with cystic fibrosis and controls. 